\title{
A multi-dynamic-factor model for stock returns
}

\author{
Victor $\mathrm{Ng}$ \\ Unicersity of Michigan, Ann Arbor, MI 48109-1234, USA \\ Robert F. Engle and Michael Rothschild \\ Unicersity of California, San Diego, La Jolla, CA 92093-0508, USA
}

\begin{abstract}
In this paper, we define dynamic and static factors and distinguish between the dynamic and static structure of asset excess returns. We examine the value-weighted market portfolio as a dynamic factor and propose an intuitively appealing procedure to search for more dynamic factors. We find evidence that the market is a dynamic factor but a three-dynamic-factor model is superior in modelling the decile portfolios. The two additional factors are correlated with a January dummy and Bond risk premium and with production growth and a recession dummy, respectively. We found that small firms are more sensitive to the January/Bond risk factor, while large firms are more sensitive to the Production/Recession factor. We found that after accounting for the systematic risk corresponding to the three dynamic factors, there is not much of a static component of asset risk premium and there is no evidence for a higher 'unexplained' return on small firm portfolios.
\end{abstract}

\section{Introduction}

The goal of asset pricing theory is to explain why different securities offer different risk premia (expected return minus a riskfree rate). In static models, the riskfree rate and the expected returns of securities are assumed to be time-invariant and hence the issue of interest is simply the cross-sectional differences in security risk premia. With mounting evidence of time-varying expected returns and time-varying variances and covariances, the level of complexity of the problem increases and so does the number of interesting questions. In the more general context, the risk premium of a security can be thought of as having a dynamic component that varies over time and a static component that is time-invariant. In this paper we ask whether the differences in the risk premia are coming mainly form the dynamic component or from the static component and what influences the dynamic and static 
components. These issues have very important implications on asset pricing and portfolio allocation.

In this paper, we introduce a multi-factor model in which there are 'dynamic factors' and 'static factors'. The dynamic factors are related to the dynamic component of the vector of asset risk premia, while the static factors are related to the static component. Based on our model, we then explicitly investigate: (1) whether the value-weighted market portfolio is a dynamic factor, (2) whether there are other dynamic factors, (3) how important is the static component relative to the dynamic component of the vector of asset risk premia, (4) whether some asset pricing anomalies can be explained by our more general dynamic factor model, and (5) what are the relationships between dynamic factors and some macro-variables.

The paper is organized as follows: after we setup the framework for analysis and define dynamic and static factors in the next section, we will define a dynamic market model as a special case of our factor structure in section 3 . In section 4 we will examine the empirical validity of the dynamic market model using a vector of excess returns from ten-decile portfolios traded in the NYSE and the AMEX. In section 5, we further explore the existence of additional dynamic factors. We introduce an intuitively appealing procedure to find dynamic factors and examine the dynamic behavior of some candidate dynamic factors. In section 6 , we estimate a three-dynamicfactor model for the set of asset excess returns based on the candidate dynamic factors found in section 5 . In section 7, we consider the relationship between our dynamic factors and some macro-variables. Section 8 concludes the paper.

\section{The framework of analysis}

\subsection{A multi-factor model with dynamic factors}

Let $\boldsymbol{y}_{t}$ be a vector of $N$ asset excess returns (rates of return minus a riskfree rate). A typical multi-factor model is

$$
\boldsymbol{y}_{t}=\boldsymbol{\mu}_{t}+\sum_{k=1}^{K} \boldsymbol{\beta}_{k} \cdot f_{k t}+\boldsymbol{\varepsilon}_{t} \text {, }
$$

where $\boldsymbol{\mu}_{t}$ is the $N \times 1$ vector of expected excess returns (or risk premia), $K$ is the total number of factors, $\boldsymbol{\beta}_{k}(k=1, \ldots, K)$ are linearly independent (nonstochastic) $N \times 1$ vectors of factor loadings, $f_{k t}(k=1, \ldots, K)$ are uncorrelated random variables called the factors, and $\varepsilon_{\imath}$ is the $N \times 1$ vector of idiosyncratic noises. 
Using an arbitrage argument similar to that in Ross (1976) or a consumption beta argument as in Engle, $\mathrm{Ng}$, and Rothschild (1990), the vector of asset risk premia can be related to a set of factor risk premia. Furthermore, as the factors are uncorrelated, it is natural to assume that the risk premium of each factor is a function of the conditional volatility of the factor itself. Hence, the vector of asset risk premia can be written as

$$
\boldsymbol{\mu}_{t}=\sum_{k=1}^{K} \boldsymbol{\beta}_{k} \cdot \pi_{k}\left(\theta_{k t}\right)
$$

where $\theta_{k t}$ is the conditional variance of factor $k$ at time $t$ and $\pi_{k}(\cdot)$ is a continuous function.

Eq. (2) suggest a very natural way to classify factors into dynamic factors and static factors and to decompose the vector of asset risk premia into a dynamic component and a static component. First, a factor with constant conditional variance would have a constant factor risk premia and hence should have no effect on the dynamic behavior of individual asset risk premium. Such a factor can be called a static factor. On the contrary, a factor with time-varying conditional variance would generally have a time-varying factor risk premium and hence should play a role in determining the time series behavior of individual asset risk premia. Such a factor can be called a dynamic factor.

Now, if we reorder the factors such that the first $K_{\mathrm{d}}$ factors are dynamic and the remaining $K-K_{\mathrm{d}}$ factors are static, then eqs. (1) and (2) can be rewritten as

$$
\begin{aligned}
& \boldsymbol{y}_{t}=\boldsymbol{\mu}_{t}+\sum_{k=1}^{K_{\mathrm{d}}} \boldsymbol{\beta}_{k} \cdot f_{k t}+\sum_{j=K_{\mathrm{d}}+1}^{K} \boldsymbol{\beta}_{j} \cdot f_{j t}+\boldsymbol{\varepsilon}_{t}, \\
& \boldsymbol{\mu}_{t}=\boldsymbol{\mu}_{t}^{\mathrm{d}}+\boldsymbol{\mu}^{\mathrm{s}},
\end{aligned}
$$

where

$$
\boldsymbol{\mu}_{t}^{\mathrm{d}} \equiv \sum_{k=1}^{K_{\mathrm{d}}} \boldsymbol{\beta}_{k} \cdot \pi_{k}\left(\theta_{k t}\right) \quad \text { and } \quad \boldsymbol{\mu}^{\mathrm{s}} \equiv \sum_{j=K_{\mathrm{d}}+1}^{K} \boldsymbol{\beta}_{j} \cdot \pi_{j}\left(\theta_{j}\right)
$$

In eq. (3b), $\mu_{t}^{\mathrm{d}}$ is the dynamic component and $\mu^{\mathrm{s}}$ is the static component of the vector of asset risk premia.

The conditional covariance matrix of asset excess returns is

$$
\operatorname{cov}_{t-1}\left(y_{t}, y_{t}\right)=\sum_{k=1}^{K_{\mathrm{d}}} \boldsymbol{\beta}_{k} \boldsymbol{\beta}_{k}^{\prime} \theta_{k t}+\boldsymbol{\Omega}
$$


where

$$
\boldsymbol{\Omega}=\sum_{j=K_{\mathrm{d}}+1}^{K} \boldsymbol{\beta}_{j} \boldsymbol{\beta}_{j}^{\prime} \theta_{j}+\boldsymbol{\Lambda}
$$

is the constant part of the conditional covariance matrix, and $\boldsymbol{\Lambda}$ is the covariance matrix of the idiosyncratic noise of the asset excess returns.

Under our structure, the dynamic behavior of the conditional covariance matrix of asset excess returns is driven solely by the dynamic behavior of the conditional volatility of the factors. If the conditional variance of the portfolios of assets that represent (or mimic) these factors follows individual GARCH processes, then the conditional covariance structure will reduce to the FACTOR-ARCH model introduced by Engle (1987) and examined by Engle, $\mathrm{Ng}$, and Rothschild (1990) for the excess returns of Treasury bills. A more detailed description of the relationship between dynamic factors and factor-representing portfolios and the relationship between the covariance structure in eq. (4) and the FACTOR-ARCH model is given in Engle, Ng, and Rothschild (1990). We do not repeat the arguments here.

\subsection{A corresponding model with time-varying factor betas}

The model described above in which some factors have time-varying volatility but all factor betas are time-invariant can also be rewritten into a model in which the factors are normalized to have unit variance but the betas of the assets with respect to the factors are time-varying. If we define

$$
\begin{array}{ll}
f_{k t}^{*}=f_{k t} / \sqrt{ } \theta_{k t}, & k=1, \ldots, K_{\mathrm{d}}, \\
f_{j t}^{*}=f_{j t} / \sqrt{ } \theta_{j}, & j=K_{\mathrm{d}}+1, \ldots, K,
\end{array}
$$

then the factor model (1) can be rewritten as

$$
\boldsymbol{y}_{t}=\boldsymbol{\mu}_{t}+\sum_{k=1}^{K_{\mathrm{d}}} \boldsymbol{g}_{k t} \cdot f_{k t}^{*}+\sum_{j=K_{\mathrm{d}}+1}^{K} \boldsymbol{b}_{j} \cdot f_{j t}^{*}+\boldsymbol{\varepsilon}_{t}
$$

where $\boldsymbol{g}_{k t}=\boldsymbol{\beta}_{k v} \theta_{k t}\left(k=1, \ldots, K_{\mathrm{d}}\right)$ are the time-varying factor betas and $\boldsymbol{b}_{j}=\boldsymbol{\beta}_{j} \theta_{j}\left(j=K_{\mathrm{d}}+1, \ldots, K\right)$ are factor betas that are constant through time.

With the new definition for the betas, the factor risk premia are

$$
\begin{array}{ll}
\pi_{k t}^{*}=\pi_{k}\left(\theta_{k t}\right) / \sqrt{ } \theta_{k t}, & k=1, \ldots, K_{\mathrm{d}}, \\
\pi_{j}^{*}=\pi_{j}\left(\theta_{j}\right) / \sqrt{ } \theta_{j}, & j=K_{\mathrm{d}}+1, \ldots, K .
\end{array}
$$


The factor model under this alternative formulation has time-varying factor betas but with the standard zero mean unit variance factors. The dynamic factors, in this context, are again factors with time-varying risk premia. Moreover, individual assets have time-varying betas with respect to the dynamic factors but not the static factors. The conditional covariance matrix of asset excess returns under this time-varying factor beta formulation is also given by eq. (4).

\section{A dynamic market model}

Many researchers have found that the value-weighted stock market portfolio has time-varying return volatility. See, for example, French, Schwert, and Stambaugh (1987), Chou (1988), or the survey in this volume by Bollerslev, Chou, and Kroner (1991). Given the role of the traditional market model (or single-index model) in the asset pricing literature, it is interesting to ask (1) whether the market portfolio is in fact a dynamic factor, (2) whether it is the only dynamic factor, and (3) is it likely that we still have some static factors after the dynamic nature of the model has been taken into account?

A multi-factor model with one dynamic factor which is the market and possibly several other static factors can be called a dynamic market model. Under the dynamic market model, the vector of excess returns can be written as

$$
\boldsymbol{y}_{t}=\boldsymbol{\mu}_{t}+\boldsymbol{\beta}_{m} \cdot f_{m t}+\sum_{j=1}^{K_{\mathrm{s}}} \boldsymbol{\beta}_{j} \cdot f_{j t}+\boldsymbol{\varepsilon}_{t}
$$

where $f_{m t}$ is the unanticipated component of the market excess return, $\boldsymbol{\beta}_{m}$ is the vector of market betas, $f_{j t}\left(j=1, \ldots, K_{s}\right)$ are the static factors, and $\boldsymbol{\beta}_{j}$ $\left(j=1, \ldots, K_{\mathrm{s}}\right)$ are vectors of factor loadings for the static factors.

Let $\pi_{m}\left(\theta_{m t}\right)$ be the time-varying market risk premium which is a function of the conditional variance of the market excess return, $\theta_{m t}$. The vector of risk premia, $\boldsymbol{\mu}_{t}$, is given by

$$
\boldsymbol{\mu}_{t}=\boldsymbol{\beta}_{m} \pi_{m}\left(\theta_{k I}\right)+\boldsymbol{\mu}^{\mathrm{s}}
$$

where $\boldsymbol{\mu}^{\mathrm{s}}$ is the static component of the vector of asset risk premia which is related to the risk premia of the static factors and the betas of the assets with respect to the static factors.

The dynamic market model also has one other very interesting property, which is that the dynamics of the conditional covariance matrix of asset returns is driven solely by the conditional volatility of the market excess 
return,

$$
\operatorname{cov}\left(\boldsymbol{y}_{t}, \boldsymbol{y}_{t}\right)=\boldsymbol{\beta}_{m} \boldsymbol{\beta}_{m}^{\prime} \boldsymbol{\theta}_{m t}+\boldsymbol{\Omega}
$$

where $\boldsymbol{\Omega}$ is the constant part of the conditional covariance matrix of asset excess returns. If $\boldsymbol{\Lambda}$ is the covariance matrix of the idiosyncratic noise of the assets, then $\boldsymbol{\Omega}$ should be

$$
\boldsymbol{\Omega}=\sum_{j=1}^{K} \boldsymbol{\beta}_{j} \boldsymbol{\beta}_{j}^{\prime} \theta_{j}+\boldsymbol{\Lambda}
$$

Schwert and Seguin (1990) independently proposed an apparently more general model which they called a single-index model of stock return heteroskedasticity. They replace (8) with

$$
\operatorname{cov}_{t-1}\left(y_{t}, y_{t}\right)=A_{0}+A_{2} \theta_{m t},
$$

where $A_{0}$ and $A_{2}$ must be symmetric positive semidefinite square matrices. Because $y_{m t}=w^{\prime} y_{t}$, for a set of market weights $w$ (which in their case are equal weights), $w^{\prime} A_{0} w=0$ and $w^{\prime} A_{2} w=1$. Rewriting $A_{0}$ in its spectral representation with (nonnegative real) eigenvalues $\lambda_{i}$ and eigenvectors $c_{i}$,

$$
A_{0}=\sum_{i=1}^{N} \lambda_{i} c_{i} c_{i}^{\prime},
$$

so that $w^{\prime} A_{0} w=0$ only if $c_{i}^{\prime} w=0$ for all nonzero $\lambda_{i}$. If follows immediately that $A_{0} w=0$. Hence, the vector of market betas,

$$
b_{m}=\operatorname{cov}_{t-1}\left(y_{t}, y_{m t}\right) / \operatorname{var}_{t-1}\left(y_{m t}\right)=\left(A_{0} w+\theta_{m t} A_{2} w\right) / \theta_{m t},
$$

is simply given by $A_{2} w$. Schwert and Seguin estimate $\left(8^{\prime}\right)$ and (10) for a vector of returns of five equally-weighted size-ranked portfolios of NYSE common stocks from 1927 to 1986 . They find evidence that the conditional variance of their size-ranked portfolios are affected by the time-varying conditional variance of the market. They also report evidence that $A_{0} w \neq 0$, which they fail to interpret as evidence against their model and instead interpret as evidence for time-varying market betas. Quite surprisingly, they also find that the small firm anomaly is stronger in the dynamic context than in a static CAPM framework. We take these latter findings as evidence that the singleindex model is too restrictive. This is consistent with the findings we report below and further motivates the search for additional dynamic factors. 


\section{Estimating and testing the dynamic market model}

\subsection{Additional model specifications and estimation method}

In order to estimate and test the dynamic market model we need to specify the dynamics of $\theta_{m t}$ and the functional form of $\pi_{m}\left(\theta_{m t}\right)$. A natural and convenient model is the GARCH-M model proposed in Engle, Lilien, and Robins (1987) which was applied to stock market data by French, Schwert, and Stambaugh (1987) and Chou (1988). Under a GARCH(1,1)-M model, $\theta_{m t}$ and $\pi_{m}\left(\theta_{m t}\right)$ are given by

$$
\begin{aligned}
& \theta_{m t}=\omega_{m}+\varphi_{m} \theta_{m t-1}+\phi_{m} u_{m t-1}^{2}, \\
& \pi_{m}\left(\theta_{m t}\right)=c_{m}+\gamma_{m} \theta_{m t},
\end{aligned}
$$

where $u_{m t} \equiv y_{m t}-\pi_{m}\left(\theta_{m t}\right)$ and $y_{m t}$ is simply the market excess return at time $t$.

The completely specified system as described by eqs. (2), (3), (7), (11a), and (11b) can in principle be estimated by full maximum likelihood over the full set of parameters: $\left\{\omega_{m}, \varphi_{m}, \phi_{m}, c_{m}, \gamma_{m}, \beta_{i m}(i=1, \ldots, N), \mu_{i}^{\mathrm{s}}(i=1, \ldots, N)\right.$, and $\left.\Omega_{i j}(i=1, \ldots, N, j=1, \ldots, N)\right\}$ assuming a certain joint distribution (presumably the conditional multivariate normal distribution) for the unpredictable part of asset excess returns. This is potentially a very large system with a large number of parameters when $N$ is large. For instance, the total number of parameters is 80 when $N$ is 10,255 when $N=20$, and 5255 when $N=100$. Given the large number of parameters (which could easily be larger than the total number of time periods available) and given the fact that there are a lot of parameters in the constant part of the conditional covariance matrix that we are not really interested in, a simpler tow-step estimation procedure might be more practical than a full maximum likelihood estimation procedure. We sacrifice efficiency but maintain consistency of the parameters of interest we estimate.

In the first step of the two-step estimation method, A univariate GARCH(1,1)-M model as described by eqs. (11a) and (11b) is fitted to the market excess return. In the second step, the estimated conditional mean and variance of the market excess return is then taken as the data series in univariate maximum likelihood estimation of the conditional mean and variance equations of each individual asset excess return series implied by eqs. (7) and (8),

$$
\begin{aligned}
& \mu_{i t}=\mu_{i}^{\mathrm{s}}+\beta_{m i} \pi_{m}\left(\theta_{m t}\right), \\
& h_{i t}=\Omega_{i i}+\beta_{m i}^{2} \theta_{m t},
\end{aligned}
$$


where $\mu_{i t}$ is the risk premium of asset $i, \mu_{i}^{\mathrm{s}}$ is the static component of the risk premium of asset $i, \beta_{m i}$ is the conditional market beta of asset $i, h_{i t}$ is the conditional excess return variance of asset $i$, and $\Omega_{i i}$ is the $i$ th diagonal element of $\boldsymbol{\Omega}$ (the constant part of the conditional covariance matrix of asset excess returns).

This two-step procedure ignores cross-asset correlations and cross-asset parameter restrictions which both sacrifice efficiency. Lin (1989) considers some approximations to full information maximum likelihood by doing one iteration full maximum likelihood after the second step. However, given the large number of parameters relative to the number of observations, the one additional step might not be feasible for a large system. A second disadvantage of the two-step method is the fact that the computed $t$-ratios do not have limiting normal distributions. Monte Carlo evidence in Lin (1989) suggests that in similar situations they may in fact be conservative. The clear advantage of the two-step estimator is the ease with which it can be expanded to apply to larger and larger systems. In fact, once the market risk premium and the market volatility are estimated, they can be used to estimate risk premia for sets of individual assets without needing to reestimate the entire system.

\subsection{Empirical results}

The data we use to evaluate the dynamic market model is a vector of monthly excess returns of ten decile portfolios of stocks traded in the NYSE and AMEX. The value-weighted NYSE + AMEX portfolio is taken as the market proxy. The monthly stock returns data are obtained from the 1985 CRSP Index Tape. The monthly returns of a one-month Treasury bill which is used as the riskfree asset in the computation of the excess returns are obtained from the Fama Term Structure File in the 1985 CRSP Government Bond Tape. The sample period is from August 1964 to November 1985. A total of 256 monthly observations.

Summary statistics for the excess returns series are given in table 1 . In the table, Dec. 1 is the portfolio of the smallest firms and Dec. 10 is the portfolio of the largest firms. The average monthly excess returns of the ten decile portfolios have a close to monotonic pattern, with the small firm portfolios having higher average monthly excess returns. The average monthly excess return of Dec. 1 (the portfolio of the smallest firms) is about $1.3 \%$ higher than the average monthly excess return of Dec. 10 (the portfolio of the largest firms). The Ljung-Box statistics (QS12) for 12th-order serial correlation in the squares of the monthly excess return series are significant at the $5 \%$ level for most of the portfolios, especially the small firm portfolios. The Ljung-Box statistics for the levels (Q12) are also very significant for the small firm portfolios. These are evidence of time-varying excess return volatilities 
Table 1

Summary statistics for individual asset cxcess returns.

\begin{tabular}{lcccccc}
\hline Assets & Mean & Var. & Skew. & Kurt. & Q12 & QS12 \\
\hline Dec. 1 & 1.4649 & 82.075 & 1.19 & 8.50 & 41.67 & 40.33 \\
Dec. 2 & 1.0329 & 61.669 & 0.70 & 7.30 & 30.26 & 31.02 \\
Dec. 3 & 1.0291 & 52.834 & 0.45 & 6.38 & 23.98 & 23.80 \\
Dec. 4 & 0.9556 & 47.498 & 0.33 & 6.03 & 18.73 & 21.19 \\
Dec. 5 & 0.7853 & 45.017 & 0.19 & 5.49 & 19.19 & 21.79 \\
Dec. 6 & 0.7979 & 38.853 & 0.12 & 5.42 & 17.41 & 14.86 \\
Dec. 7 & 0.7010 & 32.618 & -0.07 & 4.67 & 17.17 & 14.96 \\
Dec. 8 & 0.5217 & 29.833 & 0.05 & 4.49 & 17.08 & 12.02 \\
Dec. 9 & 0.4716 & 25.308 & 0.12 & 4.09 & 15.01 & 21.59 \\
Dec. 10 & 0.1443 & 17.406 & 0.17 & 4.17 & 10.58 & 30.94 \\
\hline
\end{tabular}

and time-varying risk premia. In other words, there is no doubt that there is some dynamics in the set of asset excess returns yet to be explained. 'The dynamic market model is a reasonable first candidate.

Our first-step estimation for the dynamic market model yields the following results for the $\mathrm{GARCH}(1,1)$-M model for the market excess returns (asymptotic $t$-statistics in parentheses):

$$
\begin{aligned}
& \pi_{m t}=-3.3761+0.1982 \cdot \theta_{m t}, \\
& \theta_{m t}=1.9348+0.8461 \cdot \theta_{m t-1}+0.0518 \cdot u_{m t-1}^{2}, \\
& \text { (1.68) (12.59) (1.79) }
\end{aligned}
$$

Diagnosis:

$$
\begin{array}{l|l|l|l}
\text { TSA1 }=3.19 & \text { TNA3 }=14.34 & \text { Skew. }=-0.12 & \text { Kurt. }=3.57 \\
\hline \text { Q12 = 11.64 } & \text { QS12 }=6.08 & &
\end{array}
$$

Several diagnostic statistics are also computed and reported above. They support the GARCH(1,1)-M specification for the excess return of the valueweighted market portfolio. TSA1 is the one-degree-of-freedom Lagrange Multiplier statistic testing for $u_{m t-2}^{2}$ as an additional variable in the variance equation. It is insignificant at the standard 5\% level. TNA3 is the threedegree-of-freedom likelihood ratio statistic testing the null hypothesis that the coefficients corresponding to $\theta_{m t}$ in the mean equation and $\theta_{m t-1}$ and $u_{m t-1}^{2}$ in the variance equation are zero. Although the distribution of this statistic is unknown under the null, a chi-square(3) is presumably an upper bound, hence it is highly significant. The coefficient of skewness (Skew.) and 
Table 2

A dynamic market model for individual assets. ${ }^{\text {a }}$

\begin{tabular}{|c|c|}
\hline$\mu_{t t}={ }_{(1.17)}^{0.6356}+\underset{(11.01)}{2.1781} \cdot \pi_{m t}$ & $\begin{aligned} h_{1 t}= & -16.8629+2.1718^{2} \cdot \theta_{m t}\end{aligned}$ \\
\hline$\mu_{2 t}=\underset{(0.76)}{0.3667}+\underset{(9.02)}{1.6348} \cdot \pi_{m t}$ & $h_{2 t}=\underset{(0.49)}{5.8455}+1.6348^{2} \cdot \theta_{m t}$ \\
\hline$\mu_{3 t}=\underset{(0.92)}{0.4114}+\underset{(7.96)}{1.4409 \cdot \pi_{m t}}$ & $h_{3 t}=\underset{(0.94)}{9.6825}+1.4409^{2} \cdot \theta_{m t}$ \\
\hline$\mu_{4 t}=\underset{(0.92)}{0.3955}+\underset{(7.41)}{1.3270} \cdot \pi_{m t}$ & $h_{4 t}=\underset{(1.16)}{11.0883}+1.3270^{2} \cdot \theta_{m t}$ \\
\hline$\mu_{S_{t}}=\underset{(0.60)}{0.2529}+\underset{(6.64)}{1.2737 \cdot \pi_{m t}}$ & $h_{5 t}=\underset{(1.22)}{11.8377+1.2737^{2} \cdot \theta_{m t}}$ \\
\hline$\mu_{6 t}=\underset{(0.81)}{0.3185}+\underset{(6.27)}{1.1155 \cdot \pi_{m t}}$ & $\begin{aligned} & h_{6 t}= 13.3600+1.1155^{2} \cdot \theta_{m t} \\
&(1.69)\end{aligned}$ \\
\hline$\mu_{7 t}=\underset{(0.70)}{0.2558}+\underset{(4.91)}{1.0441} \cdot \pi_{m t}$ & $h_{7 t}=\underset{(1.29)}{10.9431}+1.0441^{2} \cdot \theta_{m t}$ \\
\hline$\mu_{8 t}=\underset{(0.21)}{0.0740}+\underset{(5.46)}{1.0404} \cdot \pi_{m t}$ & $h_{8 t}=\underset{(1.06)}{8.0731}+1.0404^{2} \cdot \theta_{m t}$ \\
\hline$\mu_{9_{t}}=\underset{(0.15)}{0.0486}+\underset{(5.08)}{0.9289} \cdot \pi_{m t}$ & $h_{9 t}=\underset{(1.22)}{7.9551}+0.9289^{2} \cdot \theta_{m t}$ \\
\hline$\mu_{10 t}=\underset{(-0.79)}{-0.2031}+\underset{(5.43)}{0.9377} \cdot \pi$ & $\begin{aligned} h_{10 t}= & 0.1470+0.9377^{2} \cdot \theta_{m t} \\
& (0.02)\end{aligned}$ \\
\hline
\end{tabular}

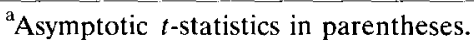

the coefficient of kurtosis (Kurt.) show no severe evidence against the conditional normality assumption. The Ljung-Box statistics for twelfth-order serial correlation in the levels (Q12) and the squares (QS12) of the normalized residuais are also insignificant at the 5\% level indicating no unexplained time variation in the conditional variance of the market excess returns.

The estimated conditional variance $\left(\theta_{m t}\right)$ and the estimated risk premium $\left(\pi_{m t}=-3.376+0.1982 \cdot \theta_{m t}\right)$ for the value-weighted market portfolio are used as predetermined variables in the second step of the estimation for the individual cxcess return processes [as given in eqs. (12a) and (12b)] of the ten decile portfolios. The estimation results are reported in table 2 .

The results are roughly as one might expect. The estimated betas decrease monotonically with firm size. However, the betas for the small firms are considerably larger than usually reported, and the intercepts, which measure abnormal returns or 'alpha', are nowhere significantly different from zero. Contrary to the Schwert and Seguin conclusion, this evidence supports the ability of the CAPM to explain the small firm excess returns. The betas estimated using a static framework are simply too small.

A test for the nonlinear restriction implied by the dynamic market model, that the beta estimated in the mean equation is the square root of the 
coefficient corresponding to the conditional variance of the market in the variance equation, is performed. The test is conducted under the artificial model:

$$
\begin{aligned}
& y_{i t}=\mu_{i}^{\mathrm{s}}+\left(\beta_{m i}+d_{m i}\right) \cdot \pi_{m t}+e_{i t}, \quad e_{i t} \mid F_{t-1} \sim \mathrm{N}\left(0, h_{i t}\right), \\
& h_{i t}=\Omega_{i i}+\beta_{m i}^{2} \cdot \theta_{m t},
\end{aligned}
$$

where $y_{i t}$ is the excess return of asset $i$ at time $t$.

Under the null hypothesis that the dynamic market model is the true data-generating mechanism, $d_{m i}$ should equal to zero. The one-degree-offreedom LM test statistics (TFR1) are reported below for each of the ten decile portfolios:

\section{Testing restrictions on mean and variance equation parameters}

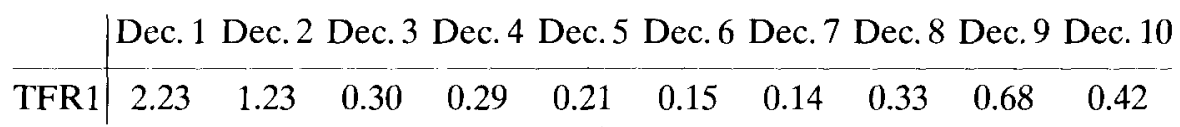

The statistics are insignificant for all ten decile portfolios at the $5 \%$ level. The results suggest that the value-weighted market portfolio might in fact be a dynamic factor. However, this result does not imply that the dynamic behavior of asset excess returns is governed solely by a single dynamic factor.

To investigate the possibility that the dynamic market model might not be able to capture completely the dynamics of asset excess returns, we consider a diagnostic test recently proposed by Engle and $\mathrm{Ng}$ (1991). We compute the normalized squared residuals for the ten decile portfolios and regress them on information from previous periods. If the heteroskedasticity is correctly modelled, the dependent variables should be independent and identically distributed and hence not predictable. The variables used to predict these residuals are the preceding six-month average of the squared residuals of decile portfolio 1 and of decile portfolio 10 . The regression equation can be expressed as

$$
\frac{e_{i t}^{2}}{h_{i t}}=\delta_{0}+\delta_{1} \sum_{j=1}^{6} e_{1 t-j}^{2}+\delta_{2} \sum_{j=1}^{6} e_{10 t-j}^{2}+v_{i t}, \quad i=1, \ldots, 10 .
$$

If the dynamic market model is correct, then the $\delta_{i}$ parameters should be zero. Hence, to test for additional dynamic structure in the data not explained by the dynamic market model, we can look at the $F$-statistics from 
these regressions. It is, however, important to note that the actual size of the test is actually smaller than the standard sizc. It is bccause the scores for the variance equation parameters in the maximum likelihood estimation of the dynamic market model are omitted from the regression. In other words, the tests are conservative.

The $F$-statistics of the above regression with degrees of freedom $(2,247)$ are reported below:

\section{Testing for unexplained dynamic structure}

\begin{tabular}{|c|c|c|c|c|c|c|c|c|c|c|}
\hline & Dec & Dec. & Dec & Dec. 4 & Dec. 5 & Dec. 6 & Dec. 7 & Dec. 8 & Dec. 9 & Dec \\
\hline & 4.82 & 4.93 & 3.72 & 4.11 & 3.76 & 3.73 & 2.16 & 2.77 & 2.23 & 2.34 \\
\hline
\end{tabular}

The $F$-statistics are significant at the standard $5 \%$ level for the first six decile portfolios. Since the test is conservative, this is a strong rejection of the dynamic market model at least for the smaller firms.

\section{More dynamic factors}

Given that the dynamic market model cannot completely capture the dynamics of the asset excess returns, it is interesting and important to examine whether these remaining differences in the dynamic components of asset excess returns can be explained by a more general dynamic factor model with more than one dynamic factor. The difficulty of such an analysis is in finding the remaining dynamic factors. Since factors are by definition mutually uncorrelated, the set of portfolios with zero conditional market beta but with changing conditional variances and changing risk premia are reasonable candidates for additional dynamic factors.

Let $w$ be a $10 \times 1$ vector with $\boldsymbol{w}^{\prime} \boldsymbol{\beta}_{m}=0$ and $\boldsymbol{w}^{\prime} \mathbf{1}=1$. If the dynamic market model given in (8) is correct, the excess return of a portfolio constructed with $\boldsymbol{w}$ as the vector of weights would have a constant conditional variance $\theta_{w} \equiv \boldsymbol{w}^{\prime} \operatorname{cov}_{t-1}\left(\boldsymbol{y}_{t}, \boldsymbol{y}_{t}\right) \boldsymbol{w}=\boldsymbol{w}^{\prime} \boldsymbol{\Omega} \boldsymbol{w}$. Because $\boldsymbol{w}^{\prime} \boldsymbol{\beta}_{m}=\mathbf{0}$, such portfolios will be called zero conditional market beta portfolios. If there are more dynamic factors, then such portfolios may have time-varying variances and means. From the set of ten decile portfolios, we construct a set of nine zero conditional market beta portfolios using restricted principal component analysis on the unconditional covariance matrix of asset excess returns. The restriction is that each of these portfolios has a zero conditional market beta and be mutually uncorrelated. Let $\mathbf{H}$ be the unconditional covariance matrix of the excess returns of the ten decile portfolios, 1 be a $10 \times 1$ vector of one's, and $\boldsymbol{w}_{j}$ be the $10 \times 1$ vector of weights for the $j$ th zero conditional 
Table 3

Weights of the zero conditional market beta portfolios.

\begin{tabular}{|c|c|c|c|c|c|c|c|c|c|}
\hline & P1 & $\mathrm{P} 2$ & P3 & P4 & P5 & P6 & $\mathrm{P7}$ & P8 & P9 \\
\hline Dec. 1 & -0.72 & 20.98 & -6.97 & -2.51 & 1.72 & 1.88 & -75.73 & 3.91 & 2.84 \\
\hline Dec. 2 & 30 & 7.54 & & & 0 & -7.72 & 368.58 & -10.78 & -27.14 \\
\hline Dec. 3 & -0.07 & 12.98 & 6.70 & -14.69 & 0.21 & 6.16 & 1080.07 & 13.48 & 72.27 \\
\hline Dec. 4 & -0.05 & 14.92 & 2.47 & -1.89 & -1.05 & 8.41 & -1943.20 & 1.88 & 90.17 \\
\hline Dec. 5 & 0.17 & 13.04 & -2.32 & -10.86 & 0.55 & -7.48 & 44.10 & -33.05 & -101.37 \\
\hline Dec. 6 & 0.27 & 13.62 & -2.97 & 2.25 & 6.65 & -44.19 & -118.70 & 23.51 & -105.92 \\
\hline Dec. 7 & 0.32 & 7.49 & -2.94 & 5.58 & 6.69 & 59.51 & 219.62 & 8.47 & -83.73 \\
\hline Dec. 8 & 0.39 & -1.58 & -4.48 & 3.34 & -29.82 & -3.03 & 345.23 & 1.60 & 59.06 \\
\hline Dec. 9 & 0.45 & -7.44 & -3.19 & 5.35 & 18.68 & -10.97 & 439.46 & -9.61 & 164.52 \\
\hline Dec. 10 & 0.44 & -38.56 & 6.92 & -3.73 & -0.28 & -1.57 & -358.43 & 1.59 & -69.70 \\
\hline
\end{tabular}

market beta portfolio $(j=1, \ldots, 9)$. The first weight vector, $\boldsymbol{w}_{1}$, is set equal to $g_{1} /\left(g_{1}^{\prime} 1\right)$, where $g_{1}$ is the solution to the restricted principal component problem: $\max _{\boldsymbol{g}} \boldsymbol{g}^{\prime} \mathbf{H} \boldsymbol{g}$ subject to $\boldsymbol{g}^{\prime} \boldsymbol{g}=1$ and $\boldsymbol{g}^{\prime} \boldsymbol{\beta}_{m}=0$. The second weight vector, $\boldsymbol{w}_{2}$, is set equal to $\boldsymbol{g}_{2} /\left(\boldsymbol{g}_{2}^{\prime} \mathbf{1}\right)$, where $\boldsymbol{g}_{2}$ is the solution to the problem: $\max _{\boldsymbol{g}} \boldsymbol{g}^{\prime} \mathbf{H} \boldsymbol{g}$ subject to $\boldsymbol{g}^{\prime} \boldsymbol{g}=1, \boldsymbol{g}^{\prime} \mathbf{H} \boldsymbol{g}_{1}=\boldsymbol{g}^{\prime} \boldsymbol{\beta}_{m}=0$. The third weight vector, $w_{3}$, is set equal to $g_{3} /\left(g_{3}^{\prime} 1\right)$, where $g_{3}$ is the solution to the problem: $\max _{\boldsymbol{g}} \boldsymbol{g}^{\prime} \mathbf{H} \boldsymbol{g}$ subject to $\boldsymbol{g}^{\prime} \boldsymbol{g}=1, \boldsymbol{g}^{\prime} \mathbf{H} \boldsymbol{g}_{1}=\boldsymbol{g}^{\prime} \mathbf{H i g}_{2}=\boldsymbol{g}^{\prime} \boldsymbol{\beta}_{\boldsymbol{m}}=0$. The weights for the remaining seven zero conditional market beta portfolios are found analogously. This procedure guarantees the production of a set of portfolios that are mutually uncorrelated and have zero conditional market betas. The portfolio weights of the set of nine zero conditional market beta portfolios are reported in table 3 .

To understand the dynamic behavior of the excess returns of these zero conditional market beta portfolios, we fit a $\operatorname{GARCH}(1,1)-\mathrm{M}$ model to the excess return of each of these portfolios. The risk premium and the conditional excess return variance of the $j$ th zero conditional market beta portfolio are denoted by $\pi_{j t}$ and $\theta_{j t}$, respectively. The estimation results are reported in table 4.

From table 4 we observe that portfolio P2 has ARCH and GARCH parameters that are both significant at the 5\% level. Portfolio P3 has a GARCH parameter which is significant at the $5 \%$ and an ARCH parameter which is significant at the $10 \%$ level. All other portfolios do not have significant ARCH parameters even though some of them have significant GARCH parameters. Since the GARCH(1,1)-M model is not well defined when the ARCH parameter is zero, these other portfolios might not really have time-varying variance. The three-degree-of-freedom likelihood ratio statistics (TN3) for constant mean and variance against the GARCH(1,1)-M and the twelve-degree-of-freedom Ljung-Box statistic for the 12th-order 
Table 4

GARCH(1,1)-M model for the zero conditional market beta portfolios. ${ }^{a}$

$$
\begin{aligned}
& \pi_{1 t}=-4.465+0.107 \cdot \theta_{1 t} \\
& \theta_{1 t}=38.27+0.009 \cdot \theta_{1 t-1}+0.006 \cdot u_{1 t-1}^{2} \\
& \pi_{2 t}=\frac{-31.51+0.002 \cdot \theta_{2 t}}{(-0.99)} \\
& \theta_{2 t}=\underset{(-0.75)}{-209.6}+\underset{(60.4)}{0.966 \cdot \theta_{2 t-1}}+\underset{(3.07)}{0.041} \cdot u_{2 t-1}^{2} \\
& \pi_{3 t}=9.9966-0.037 \cdot \theta_{3 t} \\
& \theta_{3 t}=66.44+0.722 \cdot \theta_{3 t-1}+0.069 \cdot u_{3 t-1}^{2} \\
& \text { (1.65) (4.86) (1.46) } \\
& \pi_{4 t}=-89.92+0.168 \cdot \theta_{4 t} \\
& (-0.71) \quad(0.73) \\
& \theta_{4 t}=1044.0-0.974 \cdot \theta_{4 t-1}+0.001 \cdot u_{4 t-1}^{2} \\
& \text { (11.7) }(-26.6) \\
& \text { (0.49) } \\
& \pi_{5 t}=-2.375+0.013 \cdot \theta_{5 t} \\
& \theta_{5 t}=200.3+0.362 \cdot \theta_{5 t-1}+0.121 \cdot u_{5 t-1}^{2} \\
& \text { (1.03) } \quad(0.66) \\
& \text { (1.09) } \\
& \pi_{6 t}=15.624-\quad 0.006 \cdot \theta_{6 t} \\
& (0.46) \quad(-0.37) \\
& \theta_{6 t}=\underset{(0.58)}{89.77}+\underset{(17.3)}{0.974} \cdot \theta_{6 t-1}-\underset{(-0.71)}{0.017 \cdot u_{6 t-1}^{2}} \\
& \theta_{7 t}=2\left(10^{6}\right)+0.233 \cdot \theta_{7 t-1}+0.017 \cdot u_{7 t-1}^{2} \\
& \text { (0.20) } \quad(0.06) \quad(0.21) \\
& \theta_{8 t}=930.1+0.000 \cdot \theta_{8 t-1}+0.101 \cdot u_{8 t-1}^{2} \\
& \text { (1.28) } \quad(0.00) \\
& \theta_{9 t}=22109+0.525 \cdot \theta_{9 t-1}+0.211 \cdot u_{9 t-1}^{2} \\
& \text { (1.21) (1.60) }
\end{aligned}
$$

${ }^{\mathrm{a}}$ Asymptotic $t$-statistics in parentheses.

Table 5

Diagnostic statistics for the GARCH(1,1)-M.

\begin{tabular}{lrrrrrrrrr} 
& \multicolumn{1}{r}{ P1 } & \multicolumn{1}{c}{ P2 } & \multicolumn{1}{c}{ P3 } & P4 & \multicolumn{1}{c}{ P5 } & P6 & P7 & P8 & P9 \\
\hline TN3 & 0.03 & 15.04 & 8.89 & 2.31 & 3.27 & 1.38 & 0.08 & 1.34 & 1.53 \\
QS12 & 10.35 & 5.66 & 15.33 & 4.00 & 11.51 & 8.60 & 10.13 & 0.82 & 10.76 \\
Skew. & -0.03 & -0.24 & -0.15 & 0.13 & 0.20 & -0.20 & -0.12 & 0.23 & 0.10 \\
Kurt. & 3.82 & 3.88 & 3.10 & 3.40 & 2.86 & 3.11 & 2.95 & 3.52 & 2.94 \\
\hline
\end{tabular}

serial correlation in the squared normalized residuals (QS12) reported in table 5 confirm the above observation.

The likelihood ratio statistics, IN 3 , are significant at the $5 \%$ level for both P2 and P3 but not for the other portfolios. Furthermore, the Ljung-Box statistics, QS12, are insignificant at the 5\% level for all portfolios indicating that there is no unexplained serial correlations in the conditional variance of the portfolio excess return which is not captured by the GARCH(1,1)-M. The other two statistics, Skew. and Kurt. (the coefficient of skewness and the coefficient for kurtosis for the normalized residuals), do not indicate deviation from the conditional normal assumption. 
As zero conditional market beta portfolios with time-varying conditional variances, portfolios $\mathrm{P} 2$ and $\mathrm{P} 3$ are reasonable candidates for two additional dynamic factors. We now test that the risk premium of a factor does not depend on the risk premium of any other factors and that the time-varying volatility of a factor is not driven by the time-varying volatility of any other factor. We will then use P2, P3, and the value-weighted market portfolio as three dynamic factors to explain the differences in the dynamic components of the asset risk premia.

The test is conducted as a four-degree-of-freedom likelihood ratio test adding the risk premia of two factors as explanatory variables into the mean equation of the third factor and the time-varying volatility of two factors as explanatory variables into the variance equation of the third factor. The tests are actually tests of the implications of the restrictions: $\boldsymbol{w}_{2}^{\prime} \boldsymbol{\beta}_{m}=\boldsymbol{w}_{2}^{\prime} \boldsymbol{\beta}_{3}=$ $\boldsymbol{w}_{3}^{\prime} \boldsymbol{\beta}_{m}=\boldsymbol{w}_{3}^{\prime} \boldsymbol{\beta}_{2}=\boldsymbol{w}_{m}^{\prime} \boldsymbol{\beta}_{2}=\boldsymbol{w}_{m}^{\prime} \boldsymbol{\beta}_{3}=0$ (where $\boldsymbol{w}_{m}$ is the market weight and where some but not all were imposed in the construction of the portfolios). The exact restrictions are hard to test directly as they involve cross-equation restrictions and therefore require the joint estimation of the whole system. The likelihood ratio statistics are 0.0964 (using market and P3 to explain P2), 2.2054 (using market and P2 to explain P3), and 1.8684 (using P2 and P3 to explain the market). While the limiting distributions of these statistics are unknown, they are all insignificant even at the $10 \%$ level under the chisquare(4) distribution. We have also tried adding past squared residuals of two factors as additional explanatory variables into the variance equation of the third factor. The two-degree-of-freedom likelihood statistics are 1.54 (P2 being the third factor), 5.82 ( $\mathrm{P} 3$ being the third factor), and 1.86 (the market being the third factor). They are all insignificant at the $5 \%$ level. There is no evidence of any 'causality in variance' effect between the three factors. Apparently, the dynamics of these three factors are driven by different forces. The movements between these conditional variances are also not very highly correlated. The correlation coefficients are 0.57 between the conditional variances of the market and $\mathrm{P} 2,0.35$ between the conditional variances of the market and $\mathrm{P} 3$, and 0.30 between the conditional variances of $\mathrm{P} 2$ and $\mathrm{P} 3$.

\section{A three-dynamic-factor model}

The usefulness of a three-dynamic-factor model with factors P2, P3, and the market must now be assessed. To investigate this issue, we estimate a three-dynamic-factor version of eqs. (3b) and (4) for the vector of asset excess returns using the two-step estimation method introduced in section 4. The estimated conditional variances $\theta_{m t}, \theta_{2 t}$, and $\theta_{3 t}$, and the risk premia $\pi_{m t}$, $\pi_{2 t}$, and $\pi_{3 t}$ of the three factors from the GARCH $(1,1)$-M model are used as predetermined variables to explain the dynamics of the excess returns of 
the ten decile portfolios. Maximum likelihood estimation (on the marginal distributions) using the $\mathrm{BHHH}$ algorithm gives the results as listed in table 6 .

From table 6 we observe that all conditional market betas are significant at the $5 \%$ level. Small firm portfolios have higher conditional market betas than large firm portfolios. The difference in betas is actually greater than is usually recorded from the unconditional CAPM. To illustrate the last point, we have also computed the unconditional market beta of the ten decile portfolios by regressing their excess returns on a constant and the market excess return. These unconditional market betas, $\beta_{M i}$, are reported below side-by-side with the conditional market betas, $\beta_{m i}$, from table 6 :

\section{Conditional and unconditional market betas}

\begin{tabular}{c|cccccccccc} 
& Dec. 1 & Dec. 2 & Dec. 3 & Dec. 4 & Dec. 5 & Dec. 6 & Dec. 7 & Dec. 8 & Dec. 9 & Dec. 10 \\
\hline$\beta_{M i}$ & 1.330 & 1.319 & 1.321 & 1.300 & 1.319 & 1.268 & 1.192 & 1.172 & 1.102 & 0.932 \\
$\beta_{m i}$ & 2.278 & 1.520 & 1.342 & 1.197 & 1.322 & 1.199 & 1.109 & 1.164 & 1.061 & 0.894
\end{tabular}

The conditional market betas of the small firm portfolios are substantially larger than the corresponding unconditional market betas. Also, the conditional beta is increasing with firm size at a much higher rate than the unconditional market betas.

From table 6 we also observe that small firm portfolios have higher conditional betas with respect to $\mathrm{P} 2$ than large firm portfolios and that this factor is significant at the 5\% level for the small firm portfolios. In terms of the third factor, the betas are largest for the small and large firm portfolios, but are only significant for the large firms. Finally, the constant terms in the risk premium equations which represent the static component of risk premia are not increasing in firm size. In fact, none of them are significant even at the $10 \%$ level.

The first three observations suggest that small firms are probably more risky than a traditional static CAPM would have predicted. Hence, the so-called small firm anomaly might not really be an anomaly but just a reflection of the fact that the traditional CAPM does not provide a correct risk assessment for assets. The difference between the risk premia of Dec. 1 (the portfolio of the smallest firms) and Dec. 10 (the portfolio of the largest firms) which is not captured by the dynamic factors is only $0.13 \%$, which is only one tenth of the figure from the unconditional mean excess returns. In fact, since the constant in the risk premium equation is insignificant for all ten portfolios, there might not be any difference in the risk premia which is not explained by the dynamic model. This last point also suggests that the static component of asset risk premia might not be very important relative to the dynamic component. 
Table 6

A three-dynamic-factor model for individual assets. ${ }^{a}$

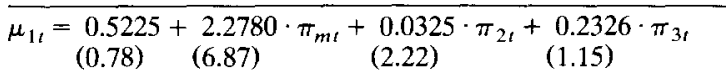

$$
\begin{aligned}
& h_{1 t}=-61.5087+2.2780^{2} \cdot \theta_{m t}+0.0325^{2} \cdot \theta_{2 t}+0.2326^{2} \cdot \theta_{3 t} \\
& (-1.79) \\
& \mu_{2 t}=\underset{(0.35)}{0.2013}+\underset{(5.36)}{1.5196} \cdot \pi_{m t}+\underset{(2.64)}{0.0293} \cdot \pi_{2 t}+\underset{(1.35)}{0.2012} \cdot \pi_{3 t} \\
& h_{2 t}=\underset{(-0.79)}{-17.1153}+1.5196^{2} \cdot \theta_{m t}+0.0293^{2} \cdot \theta_{2 t}+0.2012^{2} \cdot \theta_{3 t} \\
& \mu_{3 t}=0.1797+1.3418 \cdot \pi_{m t}+0.0240 \cdot \pi_{2 t}+0.1190 \cdot \pi_{3 t} \\
& \begin{array}{llll}
(0.31) \quad(4.81) \quad(1.91) \quad(0.80)
\end{array} \\
& h_{3 t}=\underset{(-0.04)}{-0.5949+1.3418^{2} \cdot \theta_{m t}+0.0240^{2} \cdot \theta_{2 t}+0.1190 \cdot \theta_{3 t}} \\
& \mu_{4 t}=\underset{(0.49)}{0.2514}+\underset{(4.23)}{1.1972} \cdot \pi_{m t}+\underset{(2.46)}{0.0264} \cdot \pi_{2 t}+\underset{(1.73)}{0.1904} \cdot \pi_{3 t} \\
& \begin{aligned}
h_{4 t}= & -8.2312+1.1972^{2} \cdot \theta_{m t}+0.0264^{2} \cdot \theta_{2 t}+01904^{2} \cdot \theta_{3 t} \\
(-0.51) &
\end{aligned} \\
& \mu_{S t}=\underset{(0.47)}{0.2698}+\underset{(4.77)}{1.3222} \cdot \pi_{m t}+\underset{(0.90)}{0.0140} \cdot \pi_{2 t}+\underset{(1.58)}{0.1752} \cdot \pi_{3 t} \\
& \begin{aligned}
h_{5 t}= & -3.0181+1.3222^{2} \cdot \theta_{m t}+0.0140^{2} \cdot \theta_{2 t}+0.1752^{2} \cdot \theta_{3 t} \\
(-0.19) &
\end{aligned} \\
& \mu_{6 t}=0.3883+1.1990 \cdot \pi_{m t}+00106 \cdot \pi_{2 t}+0.1709 \cdot \pi_{3 t} \\
& \begin{array}{llll}
(0.73) \quad(4.69) \quad(0.65) \quad(1.92)
\end{array} \\
& \begin{aligned}
h_{6 t}= & -1.1983+1.1990^{2} \cdot \theta_{m t}+0.0106^{2} \cdot \theta_{2 t}+0.1709^{2} \cdot \theta_{3 t} \\
(-0.09) &
\end{aligned} \\
& \mu_{7 t}=0.4082+1.1093 \cdot \pi_{m t}+0.0071 \cdot \pi_{2 t}+0.1743 \cdot \pi_{3 t} \\
& \begin{array}{llll}
(0.79) \quad(3.91) \quad(0.42) & (2.17)
\end{array} \\
& h_{7 t}=\underset{(-0.12)}{-1.5623+1.1093^{2} \cdot \theta_{m t}+0.0071^{2} \cdot \theta_{2 t}+0.1743^{2} \cdot \theta_{3 t}} \\
& \mu_{8 t}=\underset{(0.67)}{0.3721}+\underset{(4.86)}{1.1644} \cdot \pi_{m t}+\underset{(0.03)}{0.0006} \cdot \pi_{2 t}+\underset{(2.40)}{0.1827} \cdot \pi_{3 t} \\
& h_{8 t}=\underset{(-0.54)}{-7.0133}+1.1644^{2} \cdot \theta_{m t}+0.0006^{2} \cdot \theta_{2 \imath}+0.1827^{2} \cdot \theta_{3 t} \\
& \mu_{9 t}=0.4288+1.0611 \cdot \pi_{m t}-0.0012 \cdot \pi_{2 t}+0.1930 \cdot \pi_{3 t} \\
& \begin{array}{llll}
(0.85) \quad(4.77) \quad(-0.06) & (2.90)
\end{array} \\
& \begin{aligned}
h_{9 t}= & -8.8046+1.0611^{2} \cdot \theta_{m t}+0.0012^{2} \cdot \theta_{2 t}+0.1930^{2} \cdot \theta_{3 t} \\
(-0.77) &
\end{aligned} \\
& \mu_{10 t}=\underset{(1.08)}{0.3964}+\underset{(4.53)}{0.8941} \cdot \pi_{m t}-\underset{(.0 .56)}{0.0069} \cdot \pi_{2 t}+\underset{(3.51)}{0.2124} \cdot \pi_{3 t} \\
& h_{10 t}=-14.161+0.8941^{2} \cdot \theta_{m t}+0.0069^{2} \cdot \theta_{2 t}+0.2124^{2} \cdot \theta_{3 t}
\end{aligned}
$$

${ }^{a}$ Asymptotic $t$-statistics in parentheses. 
To evaluate the quality of our three-factor model and to check for misspecification, we have also explicitly tested (1) the restrictions on the mean and variance equation parameters imposed by the model, (2) the existence of unexplained serial correlation in the conditional variance of the individual asset excess returns, and (3) evidence that there remain additional dynamic factors. We have also looked for signs of violation of the conditional normality assumption. The first test is conducted under an artificial model analogous to eqs. (13a) and (13b):

$$
\begin{aligned}
y_{i t}= & \mu_{i}^{\mathrm{s}}+\left(\beta_{m i}+d_{m i}\right) \pi_{m t}+\left(\beta_{2 i}+d_{2 i}\right) \pi_{2 t} \\
& +\left(\beta_{3 i}+d_{3 i}\right) \pi_{3 t}+e_{i t}, \\
& e_{i t} \mid F_{t-1} \sim \mathrm{N}\left(0, h_{i t}\right), \\
h_{i t}= & \Omega_{i t}+\beta_{m i}^{2} \cdot \theta_{m t}+\beta_{2 i}^{2} \cdot \theta_{2 t}+\beta_{3 i}^{2} \cdot \theta_{3 \iota} .
\end{aligned}
$$

The null hypothesis is that $d_{m i}, d_{2 i}$, and $d_{3 i}$ are all zeros. The test statistic used is a three-degree-of-freedom LM test statistics and is labeled TFR3. The second test is a five-degree-of-freedom LM test for the addition of the first four lags of the own squared residuals into the variance equation of each asset and own conditional variance into the mean equation of each asset. This is actually a test for additional own ARCH-M effects. The test is an LM test of the joint zeros of coefficients $a_{1 i}, a_{2 i}, \ldots, a_{5 i}$ in the following artificial model:

$$
\begin{aligned}
y_{i t}= & \mu_{i}^{\mathrm{s}}+\beta_{m i} \cdot \pi_{m t}+\beta_{2 i} \cdot \pi_{2 t}+\beta_{3 i} \cdot \pi_{3 t}+a_{1 i} \cdot h_{i t}+e_{i t}, \\
& e_{i t} \mid F_{t-1} \sim \mathrm{N}\left(0, h_{i t}\right), \\
h_{i t}= & \Omega_{i i}+\beta_{m i}^{2} \cdot \theta_{m t}+\beta_{2 i}^{2} \cdot \theta_{2 t}+\beta_{3 i}^{2} \cdot \theta_{3 t}+\sum_{j=2}^{5} a_{j i} \cdot e_{i t-j}^{2} .
\end{aligned}
$$

The test statistic is labeled TOV5. Third, we re-estimate eq. (14) which tests for residual heteroskedasticity in the standardized residuals. Finally, to get a feel for the quality of the conditional normality assumption, we have also computed the coefficient of skewness (Skew.) and the coefficient of kurtosis (Kurt.) for the normalized residuals. All of these statistics are reported in table 7.

TFR3 is insignificant at the 5\% level for all ten decile portfolios. This indicates that the market, $\mathrm{P} 2$ and $\mathrm{P} 3$ are reasonable proxy for dynamic factors. TOV5 is also insignificant at the $5 \%$ level for all ten decile portfolios. Hence there is no own variance effect in the vector of asset excess returns. 
Table 7

Diagnostics for the three-dynamic-factor model.

\begin{tabular}{lrrrrrrrrrr}
\hline & Dec. 1 & Dec. 2 & Dec. 3 & Dec. 4 & Dec. 5 & Dec. 6 & Dec. 7 & Dec. 8 & Dec. 9 & Dec. 10 \\
\hline TFR3 & 4.55 & 3.70 & 3.46 & 2.90 & 1.63 & 1.36 & 1.24 & 1.91 & 3.80 & 1.77 \\
TOV5 & 3.67 & 5.74 & 5.31 & 6.32 & 4.19 & 5.56 & 5.08 & 2.90 & 5.35 & 0.81 \\
$F$ & 2.55 & 3.48 & 2.51 & 2.99 & 2.55 & 2.32 & 1.38 & 1.77 & 1.33 & 1.90 \\
Skew. & 0.41 & 0.11 & -0.01 & -0.09 & -0.16 & -0.19 & -0.26 & -0.18 & -0.05 & 0.09 \\
Kurt. & 4.33 & 4.52 & 4.45 & 4.17 & 4.18 & 4.31 & 4.16 & 3.74 & 3.37 & 3.54
\end{tabular}

Finally, the coefficient of skewness and the coefficient of kurtosis for the normalized residuals do not show strong disagreement with the conditional normality assumption. There is no strong evidence against the threedynamic-factor model.

\section{The factors and macro variables}

Given that P2 and P3 emerge as additional dynamic factors in the pricing of assets, it is interesting to study their relationship with some macro-variables. While a huge number of macro-variables have been considered in the literature by various works that use macro-variables in asset pricing, we have decided to focus on four variables that we think are most relevant to our application. The first, IPPNR, is the monthly percentage rate of change of the index of industrial production from the 1986 Citibase tape. Secondly, RESDUM is a recession dummy variable that takes a value of 1 when the economy is in a down turn (going from a peak to a trough in the business cycle) and 0 otherwise. The dates of troughs and peaks of business cycle are from the NBER reference dates compiled in the User's Manual for the 1986 Citibase tape. The third is $J A N D U M$, a January dummy which takes a value of 1 in the month of January and 0 otherwise. Finally, $B A A A A A$ is the difference between the annualized yields to maturity of Baa and Aaa bonds. The correlation coefficients between these macro-variables and the excess returns of the portfolios $\mathrm{P} 2$ and $\mathrm{P} 3$ are:

\section{Correlation coefficients}

\begin{tabular}{l|rrrr} 
& IPPNR & RESDUM & JANDUM & BAAAAA \\
\hline$P_{2}$ & 0.0145 & -0.0632 & 0.2417 & 0.0976 \\
$P_{3}$ & -0.0132 & -0.1548 & 0.0524 & -0.0242
\end{tabular}

The January dummy variable, $J A N D U M$, is the one that is most highly correlated with $P_{2}$. The recession dummy variable, RESDUM, is the one that 
Table 8

Regression analysis for the factors using macro-variables. ${ }^{a}$

\begin{tabular}{lccccc}
\hline $\begin{array}{l}\text { Dependent } \\
\text { variables }\end{array}$ & \multicolumn{4}{c}{ Independent variables } & \\
\hline$P_{2}$ & Const. & $I P P N R$ & $R E S D U M$ & JANDUM & BAAAAA \\
& -25.851 & 0.003 & -42.929 & 133.5 & 38.88 \\
$P_{3}$ & $(-1.16)$ & $(0.20)$ & $(-1.54)$ & $(2.95)$ & $(2.36)$ \\
& $(-2.258$ & -0.0039 & -7.7438 & 3.452 & 1.239 \\
& $(-0.78)$ & $(-1.90)$ & $(-1.99)$ & $(0.81)$ & $(0.49)$ \\
\hline
\end{tabular}

${ }^{\mathrm{a}}$ White heteroscedasticity-consistent $t$-statistics in parentheses.

is most highly correlated with $P_{3}$. For further analysis, we also regress the excess returns of $P_{2}$ and $P_{3}$ separately on a constant and the four macrovariables. The regression results are reported in table 8 .

The results in table 8 show that the excess return of $P_{2}$ is significantly related to the January dummy and the bond risk premium variable $B A A A A A$. On the other hand, the excess return of $P_{3}$ is at the margin of being significantly related to the rate of change of the index for industrial production and the recession dummy.

\section{Summary and conclusion}

In this paper we define dynamic and static factors and distinguish between the dynamic structure and the static structure of asset excess returns. We examine the value-weighted market portfolio as a dynamic factor rather than a static factor. We ask whether the market is the sole dynamic factor and propose an intuitively appealing procedure to search for more dynamic factors. We also study a dynamic, multi-factor explanation for the so-called 'small firm anomaly' and the relationship between the dynamic factors and some macro-variables.

We find evidence that the market is a dynamic factor but probably not the only one. A three-dynamic-factor model with the market as one of the dynamic factor seems to do a better job in describing the dynamic behavior of the excess returns of ten decile portfolios. Of these two additional factor, one is closely related to a January dummy and a bond risk premium variable. The other one is related to the rate of change in industrial production and a recession dummy.

We found that the small firm portfolios are more sensitive to the January/Bond risk premium actor, while the large firm portfolios are more sensitive to the Production/Recession factor. Furthermore, the conditional market betas show a much steeper monotonic relationship than the traditional unconditional market betas. We found that after accounting for the 
systematic risk corresponding to the three dynamic factors, there is not much of a static component of asset risk premium and there is no evidence for a higher 'unexplained' return on small firm portfolios. The results suggest that the static component of asset risk premia is relatively less important than the dynamic component. Also, the documented small firm anomaly might simply be a reflection of the fact that the traditional static CAPM which ignores the dynamic behavior of asset excess returns and the possible existence of additional factors does not provide a correct risk assessment for assets.

\section{References}

Baba, Y., R.F. Engle, D.K. Kraft, and K. Kroner, 1989, Multivariate simultaneous, generalized ARCH, Manuscript (University of California, San Diego, CA).

Breeden, D.T., 1979, An intertemporal asset pricing model with stochastic consumption and investment opportunities, Journal of Financial Economics 7, 265-296.

Bollerslev, T., 1986, Generalized autoregressive conditional heteroskedasticity, Journal of Econometrics 31, 307-327.

Bollerslev, T., R.F. Engle, and J.M. Wooldridge, 1988, A capital asset pricing model with time varying covariances, Journal of Political Economy 96, 116-131.

Campbell, J., 1987, Stock returns and the term structure, Journal of Financial Economics 18, 373-399.

Chamberlain, G., 1983, Funds, factors, and diversification in arbitrage pricing models, Econometrica 51, 1305-1324.

Chamberlain, G. and M. Rothschild, 1983, Arbitrage, factor structure and mean-variance analyis on large asset markets, Econometrica 51, 1281-1304.

Chou, R.Y., 1987, Volatility persistence and stock returns: Some empirical evidence using GARCH, Journal of Applied Econometrics 3, 279-294.

Diebold, F.S. and M. Nerlove, 1988, The dynamics of exhange rate volatility: A multivariate latent factor ARCH model, Journal of Applied Econometrics 4, 1-22.

Engle, R.F., 1982, Autoregressive conditional heteroscedasticity with estimates of the variance of U.K. inflation, Econometrica 50, 987-1008.

Fngle, R.F., 1987, Multivariate ARCH with factor structures: Cointegration in variance, Discussion paper no. 87-27 (Department of Economics, University of California, San Diego, CA).

Engle, R.F. and T. Bollerslev, 1986, Modelling the persistence of conditional variances, Econometric Reviews 5, 1-50.

Engle, R.F., D. Lilien, and R. Robins, 1987, Estimating time varying risk premia in the term structure: The ARCH-M model, Econometrica 55, 391-408.

Engle, R.F., V. Ng, and M. Rothschild, 1990, Asset pricing with a factor ARCH covariance structure: Empirical estimates for Treasury bills, Journal of Econometrics 45, 213-237.

Fama, E.F. and J.D. Macbeth, 1973, Kisk, return and equilibrium: Empirical tests, Journal of Political Economy LXXXI, 607-636.

Ferson, W., S. Kandel, and R. Stambaugh, 1987, Tests of asset pricing with time varying expected risk premiums and market betas, Journal of Finance 42, 201-220.

French, K.R., G.W. Schwert, and R.F. Stambaugh, 1986, Expected stock returns and volatility, Journal of Financial Economics 19, 3-29.

Hansen, L.P. and K.J. Singleton, 1983, Stochastic consumption risk aversion and the temporal behavior of stock returns, Journal of Political Economy 91, 249-265.

Keim, D. and R. Stambaugh, 1986, Predicting returns in the stock and bond markets, Journal of Financial Economics 17, 357-390.

Lin, W., 1989, Alternative estimators for factor ARCH model: A Monte Carlo comparison, Memo. (Department of Economics, University of Wisconsin, Madison, WI).

Murphy, K. and R. Topel, 1985, Estimation and inference in two step econometric models, Journal of Business and Economic Statistics 3, 370-379. 
Poterba, M.P. and L.H. Summers, 1987, The persistence of volatility and stock market fluctuations, American Economic Review 76, 1142-1151.

Rothschild, M., 1986, Asset pricing theories, in: W. Heller, R. Starr, and D. Starrett, eds., Uncertainty, information and communication - Essays in honor of Kenneth J. Arrow, Vol. III (Cambridge University Press, New York, NY).

Stambaugh, R.F., 1983, Arbitrage pricing with information, Journal of Financial Economics 12 , 357-369.

Schwert, W. and P. Seguin, Heteroskedasticity in stock returns, Journal of Finance 45, 1129-1156.

Weiss, A.A., 1982, Asymptotic theory for ARCH models: Stability, estimation and testing, Econometric Theory 2, 107-131. 